\title{
Início da mobilização em pacientes com hemorragia subaracnoidea aneurismatica
}

\author{
Start of mobilization in patients with subaracnoid \\ aneurismatic hemorrhage
}

\author{
Inicio de la movilización en pacientes con hemorragia \\ aneurismática subaracnoidea
}

\author{
Jozilane Santos Domingos ${ }^{1}$, Emília de Alencar Andrade², Rogleson \\ Albuquerque Brito $^{3}$, Aila Maria da Silva Bezerra ${ }^{4}$
}

\begin{abstract}
1.Fisioterapeuta. Residente Multiprofissional em Terapia Intensiva pela Universidade Federal do Ceará (UFC). Fortaleza - CE, Brasil. Residente Multiprofissional em Neurologia e Neurocirurgia pela Escola de Saúde Pública do Ceará (ESP/CE). Fortaleza-CE, Brasil.

2.Fisioterapeuta. Mestre em Saúde Pública pela Universidade estadual do Ceará (UECE). Preceptora do Programa de Residência Multiprofissional em Neurologia e Neurocirurgia da ESP/CE. Fisioterapeuta da Unidade de Terapia Intensiva Neurológica do Hospital Geral de Fortaleza - HGF, Fortaleza-CE, Brasil.

3Fisioterapeuta. Mestre em Ciências Médicas pela Universidade Federal do Ceará (UFC). Fortaleza-CE, Brasil.

4.Fisioterapeuta. Mestre em Saúde Coletiva pela Universidade de Fortaleza (UNIFOR). Fortaleza - CE, Brasil. Preceptora do Programa de Residência Multiprofissional em Neurologia e Neurocirurgia da ESP/CE. Fisioterapeuta da Unidade de Terapia Intensiva Neurológica do Hospital Geral de Fortaleza - HGF, Fortaleza-CE, Brasil.
\end{abstract}

\section{Resumo}

Introdução. As diretrizes para o manejo de pacientes com Hemorragia Subaracnóidea Aneurismática não apontam o momento ideal para iniciar a mobilização. Objetivo. Identificar critérios de segurança para o início da fisioterapia motora nesses pacientes. Método. Foi realizada uma revisão sistemática, desenvolvida conforme as recomendações do Preferred Reporting Intens for Systematic Reviews and Meta-Analyses (PRISMA). A busca foi feita por dois pesquisadores independentes nas bases de dados eletrônicos LILACS, SciELO, MedLine/PubMed e PEDro, utilizando a combinação dos termos: "Early Ambulation", "Early Mobilization", "Intracranial aneurysms", "Subarachnoid hemorrhage. Definidos os critérios de inclusão: artigos publicados desde o início das bases até agosto de 2020, sem restrição de idioma, que abordam sobre intervenção com fisioterapia motora e/ou sobre efeitos da quebra do repouso no paciente adulto com Hemorragia Subaracnóidea Aneurismática. Resultados. Foram incluídos nove artigos a partir dos 95 estudos encontrados na busca. Foram excluídos estudos que não atenderam aos critérios acima estabelecidos e revisões de literatura. A qualidade metodológica dos ensaios clínicos foi avaliada de acordo com a escala PEDro. O nível de evidência destes variou de quatro a cinco, sendo considerados de qualidade baixa e intermediária respectivamente. Os resultados permitiram a sumarização dos critérios de segurança em três categorias: cardiovascular, respiratória e neurológica. Nesta obtivemos maior número de itens com variáveis a serem consideradas para a elegibilidade do paciente a iniciar a mobilização. Conclusão. Os critérios de segurança concentraram-se em garantir a estabilidade neurológica e fisiológica do paciente antes do início das primeiras sessões de mobilização.

Unitermos. Mobilização precoce; Hemorragia subaracnóidea; Aneurisma cerebral; Vasoespasmo

\footnotetext{
Abstract

Introduction. The guidelines for the management of patients with Aneurysmatic Subarachnoid Hemorrhage do not indicate the ideal time to start mobilization. Objective. To identify safety criteria for the initiation of motor physiotherapy in these patients. Method. A systematic review was carried out, developed according to the recommendations of the Preferred Reporting Intens for Systematic Reviews and Meta-Analyzes (PRISMA). The search was carried out by two independent researchers in the electronic databases LILACS, SciELO,
} 
MedLine/PubMed and PEDro, using the combination of the terms: "Early Ambulation", "Early Mobilization", "Intracranial aneurysms", "Subarachnoid hemorrhage. Inclusion criteria were defined: articles published from the beginning of the bases until August 2020, without language restriction, that address intervention with motor physiotherapy and/or the effects of breaking rest in adult patients with Aneurysmatic Subarachnoid Hemorrhage. Results. Nine articles were included from the 95 studies found in the search. Studies that did not meet the criteria established above and literature reviews were excluded. The methodological quality of clinical trials was assessed according to the PEDro scale. The level of evidence of these ranged from 4 to 5, being considered of low and intermediate quality respectively. The results allowed the summary of safety criteria in three categories: cardiovascular, respiratory, and neurological. In the last category, we obtained a greater number of items with variables to be considered for the patient's eligibility to initiate mobilization. Conclusion. Safety criteria focused on ensuring the patient's neurological and physiological stability before the start of the first mobilization sessions.

Keywords. Early mobilization; Subarachnoid hemorrhage; Brain aneurysm; Vasospasm

\section{RESUMEN}

Introducción. Las pautas para el manejo de pacientes con hemorragia subaracnoidea aneurismática no indican el momento ideal para iniciar la movilización. Objetivo. Identificar criterios de seguridad para el inicio de fisioterapia motora en estos pacientes. Método. Se realizó una revisión sistemática, desarrollada de acuerdo con las recomendaciones del Preferred Reporting Intens for Systematic Reviews and Meta-Analyzes (PRISMA). La búsqueda fue realizada por dos investigadores independientes en las bases de datos electrónicas LILACS, SciELO, MedLine/PubMed y PEDro, utilizando la combinación de los términos: "Ambulación temprana", "Movilización temprana", "Aneurismas intracraneales", "Hemorragia subaracnoideaSe definieron criterios de inclusión: se incluyeron artículos publicados desde el inicio de las bases hasta agosto de 2020, sin restricción de idioma, que abordan la intervención con fisioterapia motora y/o los efectos de la interrupción del reposo en pacientes adultos con Hemorragia Subaracnoidea Aneurismática. Resultados. 9 artículos de los 95 estudios encontrados en la búsqueda. Se excluyeron los estudios que no cumplieron con los criterios establecidos anteriormente y las revisiones de la literatura. La calidad metodológica de los ensayos clínicos se evaluó según la escala PEDro. El nivel de evidencia de estos osciló entre 4 y 5, considerándose de calidad baja e intermedia respectivamente. Los resultados permitieron resumir los criterios de seguridad en tres categorías: cardiovascular, respiratoria y neurológica. En este, obtuvimos un mayor número de ítems con variables a considerar para la elegibilidad del paciente para iniciar la movilización. Conclusión. En definitiva, los criterios de seguridad se centraron en asegurar la estabilidad neurológica y fisiológica del paciente antes del inicio de las primeras sesiones de movilización.

Palabras clave: Movilización temprana; Hemorragia subaracnoidea; Aneurisma Cerebral; Vasoespasmo

Trabalho realizado na Instituição Escola de Saúde Pública do Ceará (ESP/CE). Fortaleza-CE, Brasil.

Endereço para correspondência: Jozilane Santos Domingos. R. Gustavo Sampaio 1383. Fortaleza-CE, Brasil. CEP: 60.455-001. Email: jozilanesd@gmail.com

\section{INTRODUÇÃO}

A Hemorragia Subaracnóidea aneurismática (HSAa) é um evento clínico grave caracterizado pela presença de sangue no espaço subaracnóideo, localizado entre as meninges dura-máter e aracnoide - membranas que 
revestem o Sistema Nervoso Central (SNC), proveniente do rompimento de aneurismas de vasos contidos nesse espaço ou adjacentes a ele junto a dura-máter ${ }^{1}$. É alta a taxa de mortalidade nos indivíduos com HSA, chega a alcançar os $40 \%$, sendo esta responsável por $5 \%$ de todos os eventos de disfunção neurológica com manifestação de quadro clínico súbito ${ }^{2,3}$.

As manifestações clínicas, em geral, são: cefaleia intensa e súbita, alterações do nível de consciência, vômitos, convulsões, alterações da frequência cardíaca e pressão arterial, além de déficits motores a depender do nível de acometimento do $\mathrm{SNC}^{4}$. Estes e os demais sintomas podem ser novos ou recorrentes, no caso de ressangramentos e respostas secundárias como o vasoespasmo (VE) e isquemia cerebral $^{4,5}$.

Estudos mostraram que sem a oclusão do aneurisma seja por tratamento antifibrinolítico ou cirúrgico, aproximadamente $30 \%$ dos pacientes desenvolvem ressangramento dentro de um mês após a hemorragia inicial e cerca de $50 \%$ vão a óbito ${ }^{3}$, sendo o período de maior risco de ressangramento entre duas a quatro semanas após o início dos sintomas de $\mathrm{HSAa}^{3,5}$. Uma das intervenções básicas, em teoria, para evitar o ressangramento seria o repouso total de quatro a seis semanas, no entanto, não existem evidências suficientes a favor ou contra a permanência no leito por pelo menos quatro semanas após o início dos sintomas em casos de aneurismas não tratáveis ${ }^{5,6}$. 
Considerando a particularidade do perfil de pacientes com HSAa, no contexto de sua gravidade, a fisioterapia motora, assim como para pacientes agudamente hospitalizados por outras patologias, deve ser pautada em critérios de segurança7. Os achados da literatura revelam que o repouso no leito afeta negativamente os sistemas musculoesquelético, cardiovascular, respiratório e imunológico7. No entanto, para recomendar a mobilização em pacientes que sofreram HSAa, deve-se levar em conta fatores que podem afetar a capacidade funcional desses pacientes. Tanto a monitorização contínua relacionada a tolerância à mobilização, como a análise do histórico médico, a estabilidade cardiovascular, dentre outras variáveis, devem ser consideradas ${ }^{4,8}$.

A reabilitação precoce tem mostrado eficácia em uma série de distúrbios neurológicos agudos ${ }^{9}$, no entanto, não é estabelecida ainda como parte das diretrizes de tratamento após HSAa 6 . É provável que, em parte, isso se deva ao medo de agravamento ou aumento do risco de desenvolvimento do VE cerebral com isquemia tardia, complicação mais temida. Dessa forma, as diretrizes para o manejo de pacientes com HSAa não apontam o momento ideal e com segurança para se iniciar a mobilização desses pacientes ${ }^{6,10}$.

Portanto, a identificação de critérios de segurança para o início da fisioterapia motora no perfil de paciente em estudo a partir de uma revisão sistemática, poderá trazer evidências consistentes a serem incorporadas à rotina dos serviços a fim de guiar a prática clínica e direcionar a conduta 
do fisioterapeuta ajudando-o a eleger de forma criteriosa o paciente para o início da mobilização de forma eficiente e segura. 0 presente estudo poderá direcionar o estabelecimento de critérios para um grupo específico de pacientes neurológico atendendo as suas necessidades, além de fomentar a prática clínica baseada em evidências.

Considerando o exposto acima, pretende-se, por meio de uma revisão sistemática da literatura, identificar critérios de segurança para o início da fisioterapia motora em pacientes com hemorragia subaracnóidea aneurismática.

\section{MÉTODO}

Estudo de caráter exploratório e descritivo realizado através de uma revisão sistemática, conforme as recomendações do Preferred Reporting Intens for Systematic Reviews and Meta-Analyses (PRISMA) ${ }^{11}$. Feito a partir da pergunta norteadora "Quais os critérios de segurança para o início da fisioterapia motora em pacientes com hemorragia subaracnóidea aneurismática?" Por meio da estratégia PICO $^{11}$ descrita no Quadro 1.

Quadro 1. Estratégia PICO para estruturar a pergunta norteadora ${ }^{11}$.

\begin{tabular}{|ll|}
\hline PICOS & DESCRIÇÃo \\
\hline População: & Pacientes com HSAa \\
Intervenção: & Fisioterapia motora \\
Comparação: & Repouso ou sem comparação \\
Outcome (Desfecho): & Eventos adversos \\
Study type (Tipo de estudo): & Experimentais ou não, exceto revisões. \\
\hline
\end{tabular}


A busca foi realizada por dois pesquisadores independentes no período de junho a agosto de 2020 nas bases de dados eletrônicos LILACS, SciELO, MedLine/PubMed e PEDro. Foram utilizados os termos: "Early Ambulation", "Early Mobilization", "Intracranial aneurysms", "Subarachnoid hemorrhage", segundo os Descritores da Medical Subject Headings (MeSH) e Ciência da Saúde (DeCS) cruzados por meio dos operadores boleanos. Considerando a especificidade do formato de busca na base PEDro, foram utilizados os termos: "brain aneurysm", "neurology", "cerebral vasospasm" e "subarachnoid hemorrhage" associados um por vez ao termo "early mobilization" nos espaços de busca avançada. Foram definidos como critérios de inclusão: artigos publicados desde o início das bases até o momento da busca, sem restrição de idioma, que abordam sobre intervenção com fisioterapia motora e/ou sobre efeitos da quebra do repouso no paciente adulto com HSAa. Foram excluídos estudos que não atenderam aos critérios acima estabelecidos, revisões de literatura e os duplicados na busca. A seleção dos estudos se deu pela leitura inicial por parte dos dois pesquisadores, de forma independente, dos títulos e resumos, além da leitura na íntegra das publicações nos casos em que os títulos e os resumos não foram suficientes para determinar a seleção, por fim, elegendo-se os artigos para este estudo. A qualidade metodológica dos ensaios clínicos foi avaliada de acordo com a escala Physiotherapy Evidence Database (PEDro) ${ }^{12}$, por meio de 11 itens a esta função destinados. Atribuindo aos valores de 7 
a 10 como estudos de alta qualidade; 5 e 6, de qualidade intermediária, e de zero a 4 , de baixa qualidade ${ }^{13}$. Foram extraídos os dados de interesse ao objetivo do estudo, a partir daí organizados e apresentados em tabelas.

\section{RESULTADOS}

De acordo com a busca realizada, foram encontrados inicialmente 95 artigos com base nos critérios de inclusão estabelecidos, sendo elegíveis para inclusão na amostra final deste estudo um total de 9 artigos, conforme sumarizado na Figura 1.

Figura 1. Fluxograma de Seleção dos Artigos.

N. de artigos identificados em bancos de dados eletrônicos e busca manual: 95

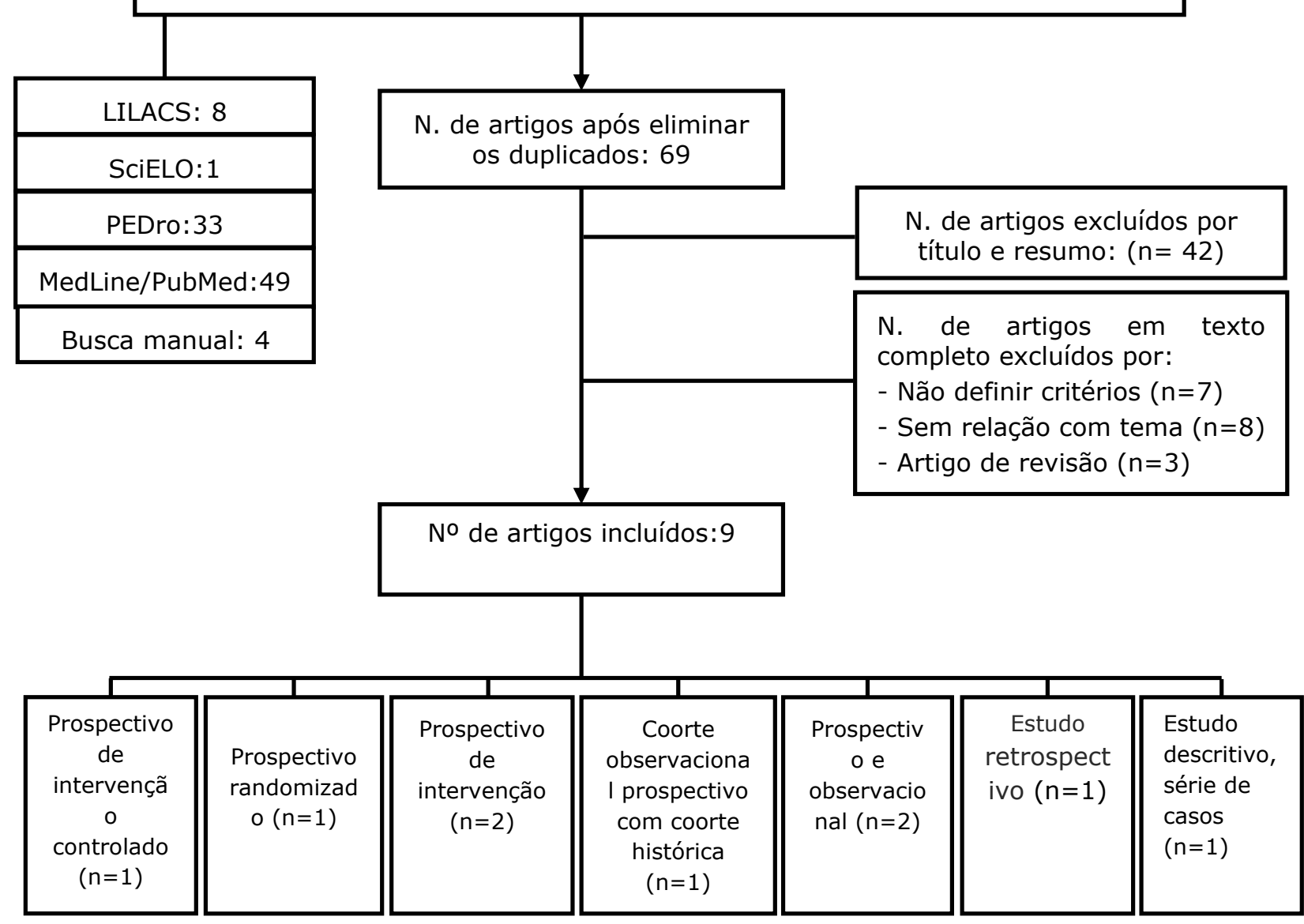


A qualidade metodológica dos estudos experimentais desta revisão está disposta na Tabela 1 . Veremos que apenas $4(44,4 \%)$ dos 9 foram passiveis de avaliação pela escala PEdro, pois os demais não são estudos de intervenção. 0 nível de evidência (NE) variou entre 4 e 5 sendo que apenas $3(75 \%)$ receberam pontuação 5 . Considerando, portanto, a pontuação máxima de 10 da escala utilizada, obtivemos 1 estudo de baixa qualidade $(\mathrm{NE}=4), 3$ de qualidade intermediaria $(N E=5)$ e nenhum de alta qualidade (NE de 7 a 10).

Tabela 1. Classificação metodológica dos artigos de acordo com a escala PEDro.

\begin{tabular}{|c|c|c|c|c|}
\hline Critérios & Karicl $2017^{14}$ & Karic $2016^{15}$ & Rocca $2016^{16}$ & Moyer $2017^{17}$ \\
\hline Critérios de elegibilidade* & Sim & Sim & Sim & Sim \\
\hline Seleção randomizada & Não & Não & Sim & Não \\
\hline Alocação secreta & Não & Não & Sim & Não \\
\hline Homogeneidade pré-tratamento & Sim & Sim & Não & Sim \\
\hline Sujeitos cegos & Não & Não & Não & Não \\
\hline Terapeutas cegos & Não & Não & Não & Não \\
\hline Avaliadores cegos & Não & Não & Não & Não \\
\hline Acompanhamento adequado & Sim & Sim & Sim & Sim \\
\hline Intenção de tratamento & Não & Sim & Não & Sim \\
\hline Comparação entre grupos & Sim & Sim & Sim & Sim \\
\hline $\begin{array}{l}\text { Medidas pontuais e de } \\
\text { variabilidade }\end{array}$ & Sim & Sim & Sim & Sim \\
\hline Total & $4 / 10$ & $5 / 10$ & $5 / 10$ & $5 / 10$ \\
\hline
\end{tabular}

*O item critérios de elegibilidade não é somado na pontuação final

A seguir, temos um panorama de todos os artigos incluídos (Tabela 2). Vemos que $6(66,6 \%)$ estudos foram desenvolvidos em UTI (neurológicas e neurocirúrgicas) e 3 $(33,3 \%)$ em enfermarias (unidades neurointermediárias). 0 país no ranking de publicações foi EUA com 4 estudos $(44,4 \%)$, seguido da Noruega com 3(33,3\%) e Suíça e Chile 
com um estudo para cada. Quanto ao ano de publicação, o mais atual foi 2019 (com apenas 1 artigo) e o mais anterior foi 2013. O tamanho amostral variou de 21 a 171 sujeitos. Pelo menos em seis estudos foi pontuado como limitação pequeno tamanho da amostra. Nem todos os artigos apresentaram com clareza os protocolos utilizados. Mas em geral, foram aplicados protocolos similares em relação aos itens e a linha de progressão partindo de elevação de cabeceira, sedestação no leito evoluindo até a deambulação. A Tabela 2 sumariza eventos adversos (EA) ocorridos e resultados relevantes do estudo.

A Tabela 3 apresenta os critérios de segurança utilizados para considerar o paciente apto ou não à MB. Seja para iniciar, continuar ou interromper a MB. Com os achados, foi possível categorizar os critérios em cardiovasculares, respiratórios, neurológicos e outros.

\section{DISCUSSÃO}

Com base nos dados dos estudos incluídos nesta revisão identificamos que critérios para assegurar o início da fisioterapia motora não são estritamente padronizados, a elegibilidade dos pacientes para mobilização (MB) deve ser avaliada diariamente considerando inúmeros aspectos ${ }^{18}$. 
Tabela 2. Referências, delineamento dos estudos, setor, objetivo, número e grupos, protocolos de mobilização, eventos adversos, resultados e limitações.

\begin{tabular}{|c|c|c|c|c|c|c|c|c|}
\hline $\begin{array}{c}\text { Referência/ } \\
\text { País }\end{array}$ & $\begin{array}{c}\text { Tipo } \\
\text { de } \\
\text { estudo }\end{array}$ & Setor & Objetivo & $\begin{array}{c}\mathbf{N}^{\circ} / \\
\text { Grupos }\end{array}$ & $\begin{array}{l}\text { Protocolo de } \\
\text { mobilização }\end{array}$ & $\begin{array}{l}\text { Eventos } \\
\text { adversos }\end{array}$ & $\begin{array}{l}\text { Resultados } \\
\text { relevantes }\end{array}$ & Limitações \\
\hline $\begin{array}{c}\text { Karic } 2017^{14} \\
\text { Noruega }\end{array}$ & $\begin{array}{c}\text { Prospec } \\
\text { tivo de } \\
\text { interve } \\
\text { nção }\end{array}$ & ENI & $\begin{array}{l}\text { Avaliar o efeito } \\
\text { da reabilitação } \\
\text { e MP nas } \\
\text { complicações } \\
\text { durante a fase } \\
\text { aguda e nos } \\
90 \text { dias após a } \\
\text { HSAa }\end{array}$ & $\begin{array}{l}\mathrm{N}=171 \\
\mathrm{GC} \text { (TTO } \\
\text { padrão) } \\
\mathrm{N}=77 ; \mathrm{GI} \\
\text { (MP além } \\
\text { do TTO } \\
\text { padrão) } \\
\mathrm{N}=94\end{array}$ & $\begin{array}{l}0-\mathrm{RL} \text { e } \mathrm{ELC} \\
30^{\circ} ; 1-\mathrm{RL} \text { e } \\
\text { ELC } 60^{\circ} ; 2-\mathrm{RL} \\
\text { e ELC } 80^{\circ} ; 3- \\
\mathrm{SD} \text { na cama; } \\
4-\mathrm{SD} \quad \text { na } \\
\text { cadeira; 5-De } \\
\text { pé e caminhar } \\
\text { ao lado da } \\
\text { cama; 6- } \\
\text { Caminhar para } \\
\text { o banheiro e } \\
\text { no corredor. }\end{array}$ & $\begin{array}{l}\text { O VE } \\
\text { moderado não } \\
\text { atrasou a MB. } \\
\text { Em caso de VE } \\
\text { grave e / ou } \\
\text { clínico, a MB } \\
\text { foi } \\
\text { interrompida } \\
\text { ou voltou para } \\
\text { a Etapa } 0 . \\
\text { Obs: Nenhum } \\
\text { PCT sofreu } \\
\text { queda } \\
\text { acidental, nem } \\
\text { remoção de } \\
\text { tubos ou linhas } \\
\text { durante a MP. }\end{array}$ & $\begin{array}{l}\text { O GI teve MB a } \\
\text { partir do } 1^{\circ} \text { dia } \\
\text { após a CDA. O } \\
\text { grau mais alto e } \\
\text { significativo de } \\
\text { MB no GI não } \\
\text { aumentou as } \\
\text { complicações. O } \\
\text { VE cerebral } \\
\text { clínico não foi } \\
\text { tão frequente no } \\
\text { GI e tendeu a } \\
\text { ser menos } \\
\text { grave. Cada } \\
\text { etapa de MB } \\
\text { alcançada } \\
\text { durante o os } \\
\text { primeiros } 4 \text { dias } \\
\text { após O CDA } \\
\text { reduziu o risco } \\
\text { de VE grave em } \\
30 \% \text {. }\end{array}$ & $\begin{array}{l}\text { Foram } \\
\text { excluídos os } \\
\text { PCTs de UTI, } \\
\text { ou seja, os } \\
\text { que tinham } \\
\text { pior condição } \\
\text { clínica e mais } \\
\text { chances de } \\
\text { complicaçõe } \\
\text { s. }\end{array}$ \\
\hline $\begin{array}{c}\text { Karic } 2016^{15} \\
\text { Noruega }\end{array}$ & $\begin{array}{l}\text { Prospec } \\
\text { tivo e } \\
\text { control } \\
\text { ado de } \\
\text { interve } \\
\text { nção }\end{array}$ & ENI & $\begin{array}{l}\text { Avaliar } \\
\text { impacto da } \\
\text { mobilização e } \\
\text { RP } \\
\text { resultado no } \\
\text { funcional } \\
\text { global um ano } \\
\text { após a HSAa. }\end{array}$ & $\begin{array}{l}\mathrm{N}=168 \\
\mathrm{GC} \text { (TTO } \\
\text { padrão) } \\
\mathrm{N}=76 / \mathrm{GI} \\
\text { (MP além } \\
\text { do TTO } \\
\text { padrão) } \\
\mathrm{N}=92\end{array}$ & $\begin{array}{l}\text { MB } \\
\text { progressiva } \\
\text { desde o RL até } \\
\text { de pé e } \\
\text { caminhando, } \\
\text { além do TTO } \\
\text { padrão da } \\
\text { unidade. }\end{array}$ & Sem registro & $\begin{array}{l}\text { Progressão mais } \\
\text { rápida da MB no } \\
\text { GI; } \\
\text { Um no } \\
\text { significativamen } \\
\text { te maior de PCTs } \\
\text { no grupo de RP } \\
\text { foram MB para a } \\
\text { beira da cama } \\
\text { ou para uma } \\
\text { cadeira no dia } 4 \\
\text { e no dia } 7 \text { após a } \\
\text { CDA; A RP não } \\
\text { foi prejudicial; }\end{array}$ & $\begin{array}{l}\text { Sem } \\
\text { randomizaçã } \\
\text { o dos PCTS. } \\
\text { Não inclui } \\
\text { PCTs de } \\
\text { quadro } \\
\text { clínico mais } \\
\text { crítico. }\end{array}$ \\
\hline $\begin{array}{l}\text { Rocca } 2016^{16} \\
\text { Suíça }\end{array}$ & $\begin{array}{l}\text { Estudo } \\
\text { prospec } \\
\text { tivo } \\
\text { random } \\
\text { izado }\end{array}$ & $\begin{array}{l}\text { UTI ou } \\
\text { UTNI }\end{array}$ & $\begin{array}{l}\text { Observar e } \\
\text { quantificar } \\
\text { mudanças na } \\
\text { atividade } \\
\text { simpática com } \\
\text { mudanças } \\
\text { posturais } \\
\text { graduais e } \\
\text { apenas com } \\
\text { movimentos } \\
\text { dos MMII após } \\
\text { RL } \\
\text { prolongado. }\end{array}$ & $\begin{array}{l}\mathrm{N}=30 \\
3 \text { grupos } \\
\text { com } \\
\text { diferente } \\
\mathrm{s} \\
\text { protocolo } \\
\mathrm{s} \text { de MB. }\end{array}$ & $\begin{array}{l}\text { PT padrão } \\
\text { (com } \\
\text { fisioterapeutas } \\
\text { ); PT com robô } \\
\text { de } \\
\text { verticalização } \\
\text { (Erigo } @ \text { ) PT } \\
\text { com } \\
\text { treinamento } \\
\text { por um } \\
\text { ergômetro de } \\
\text { MMII } \\
\text { (MOTOmed- } \\
\text { letto } \&)\end{array}$ & $\begin{array}{l}\text { Prevalência de } \\
\text { eventos } \\
\text { hipotensivos } \\
\text { no grupo de } \\
\text { PCTs MB } \\
\text { apenas por } \\
\text { fisioterapeutas } \\
\text {; } 6 \text { PCTs do } \\
\text { grupo } 1 \text { com } \\
\text { hipotensão } \\
\text { contra } 2 \text { PCTs } \\
\text { em cada um } \\
\text { dos outros } 2 \\
\text { grupos. }\end{array}$ & 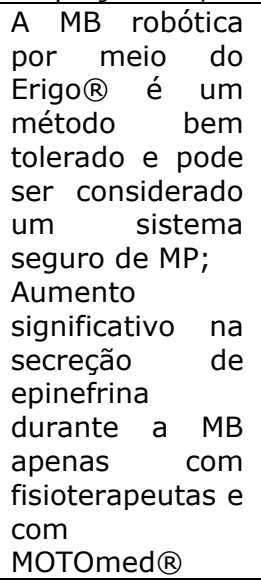 & $\begin{array}{l}\text { Heterogenei } \\
\text { dade e } \\
\text { número } \\
\text { pequeno da } \\
\text { amostra. }\end{array}$ \\
\hline $\begin{array}{c}\text { Moyer } 2017^{17} \\
\text { EUA }\end{array}$ & $\begin{array}{l}\text { Estudo } \\
\text { prospec } \\
\text { tivo de } \\
\text { interve } \\
\text { nção }\end{array}$ & UTNI & $\begin{array}{lr}\begin{array}{l}\text { Avaliar } \\
\text { viabilidade, }\end{array} \\
\text { segurança } \\
\text { resultado de } \\
\text { um PT } \\
\text { MP para PCTs } \\
\text { com HSA com } \\
\text { DVE. }\end{array}$ & $\begin{array}{l}\mathrm{N}=45 \\
\mathrm{GI} \\
\text { Prospecti } \\
\text { vo } \\
(\mathrm{N}=26) ; \\
\mathrm{GC} \\
\text { Histórico( } \\
\mathrm{N}=19)\end{array}$ & $\begin{array}{l}\text { Sentar-se na } \\
\text { beira da cama; } \\
\text { Bipedestação; } \\
\text { Passos } \\
\text { laterais; } \\
\text { AVDs. }\end{array}$ & $\begin{array}{lr}\text { De } & 101 \\
\text { sessões de } \\
\text { MB, } \\
\text { abortadas por: } \\
\text { aumento da } \\
\text { letargia (1), } \\
\text { dor (1), HIC } \\
(1), \quad \text { mau } \\
\text { funcionamento } \\
\text { do dreno (1) e } \\
\text { hipotensão } \\
\text { (2); }\end{array}$ & $\begin{array}{l}\text { Não houve } \\
\text { deslocamentos } \\
\text { de cateteres; } \\
\text { O dia da } 1^{a} \text { MB } \\
\text { foi } \\
\text { significativamen } \\
\text { te mais cedo no } \\
\text { grupo pós- } \\
\text { intervenção; }\end{array}$ & $\begin{array}{l}\text { Grupo } \\
\text { controle } \\
\text { histórico }\end{array}$ \\
\hline
\end{tabular}




\begin{tabular}{|c|c|c|c|c|c|c|c|c|}
\hline $\begin{array}{l}\text { Referência/ } \\
\text { País }\end{array}$ & $\begin{array}{c}\text { Tipo } \\
\text { de } \\
\text { estudo }\end{array}$ & Setor & Objetivo & $\begin{array}{c}\mathbf{N}^{\circ} / \\
\text { Grupos }\end{array}$ & $\begin{array}{l}\text { Protocolo de } \\
\text { mobilização }\end{array}$ & $\begin{array}{l}\text { Eventos } \\
\text { adversos }\end{array}$ & $\begin{array}{l}\text { Resultados } \\
\text { relevantes }\end{array}$ & Limitações \\
\hline $\begin{array}{c}\text { Young } 2019^{18} \\
\text { EUA }\end{array}$ & $\begin{array}{l}\text { Estudo } \\
\text { de } \\
\text { coorte } \\
\text { observa } \\
\text { cional } \\
\text { prospec } \\
\text { tivo } \\
\text { com } \\
\text { uma } \\
\text { coorte } \\
\text { histórica }\end{array}$ & $\begin{array}{c}\text { UTI } \\
\text { neuroló } \\
\text { gica }\end{array}$ & $\begin{array}{l}\text { Fazer } \quad \text { a } \\
\text { transição de } \\
\text { uma cultura de } \\
\text { repouso } \\
\text { absoluto para } \\
\text { um protocolo } \\
\text { de MB guiada } \\
\text { por TF e TO e, } \\
\text { para um } \\
\text { protocolo de MB } \\
\text { por enfermeiros }\end{array}$ & $\begin{array}{l}\mathrm{N}=56 \\
\text { Fase } \quad 0: \\
\text { sem MB } \\
(\mathrm{N}=15) ; \\
\text { Fase 1: } \\
\text { orientado } \\
\text { por TF } \\
(\mathrm{N}=24) \\
\text { Fase 2: } \\
\text { por } \\
\text { enfermeir } \\
\text { os }(\mathrm{N}=17)\end{array}$ & $\begin{array}{l}\text { MB foi definida } \\
\text { como qualquer } \\
\text { atividade do } \\
\text { PCT } \\
\text { ocorresse que } \\
\text { sentado na } \\
\text { beira da cama } \\
\text { ou qualquer } \\
\text { atividade física } \\
\text { fora da cama. }\end{array}$ & 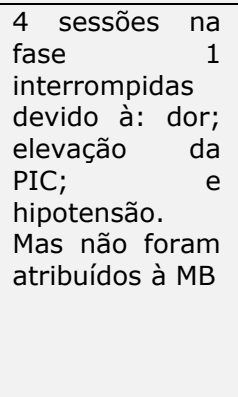 & $\begin{array}{l}\text { Nenhum } \\
\text { deslocamento de } \\
\text { cateter } \\
\text { complicações de } \\
\text { sangramento } \\
\text { foram atribuíveis } \\
\text { ao PT; } \\
\text { Sem quedas, e } \\
\text { sem } \\
\text { deslocamento } \\
\text { acidental de } \\
\text { dispositivos. }\end{array}$ & $\begin{array}{l}\text { Coleta } \\
\text { retrospectiva } \\
\text { de dados para } \\
\text { a fase 0; } \\
\text { Registro } \\
\text { inconsistente } \\
\text { dos dados: } \\
\text { duração das } \\
\text { sessões e } \\
\text { manobras de } \\
\text { MB; Amostra } \\
\text { pequena. }\end{array}$ \\
\hline $\begin{array}{c}\text { Olkowski } \\
2013^{19} \\
\text { EUA }\end{array}$ & $\begin{array}{l}\text { Estudo } \\
\text { retrospe } \\
\text { ctivo }\end{array}$ & $\begin{array}{c}\text { UTI } \\
\text { neuroci } \\
\text { rúrgica }\end{array}$ & $\begin{array}{l}\text { Determinar a } \\
\text { segurança e a } \\
\text { viabilidade de } \\
\text { um programa } \\
\text { de MP para } \\
\text { PCTs com } \\
\text { HSAa. }\end{array}$ & $\begin{array}{l}\mathrm{N}=25 \\
\text { Sem } \\
\text { grupos. }\end{array}$ & $\begin{array}{l}\text { Posicionamento } \\
\text { no leito e MB } \\
\text { passiva; ELC; } \\
\text { Sedestação; } \\
\text { Ortostatismo; } \\
\text { Deambulação } \\
\text { outros. Duração } \\
\text { de } 30 \text { a } 60 \text { min. }\end{array}$ & $\begin{array}{l}\text { Nas primeiras } \\
\text { sessões, casos } \\
\text { de: PAM <70 } \\
\text { e> } 120 \mathrm{mmHg} ; \\
\text { FC <40 ou> } \\
130 \quad \text { bpm; } \\
\text { SatO2 <88\%; } \\
\text { PIC }>15 \mathrm{~mm} \\
\mathrm{Hg} ;\end{array}$ & 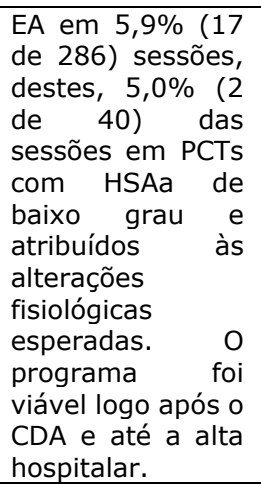 & $\begin{array}{l}\text { Estudo } \\
\text { retrospectivo, } \\
\text { sem } \\
\text { comparação } \\
\text { de } \\
\text { resultados; } \\
\text { Amostra } \\
\text { pequena. }\end{array}$ \\
\hline $\begin{array}{l}\text { Merino } 2014^{20} \\
\text { Chile }\end{array}$ & $\begin{array}{l}\text { Estudo } \\
\text { descritiv } \\
\text { o, série } \\
\text { de } \\
\text { casos }\end{array}$ & $\begin{array}{c}\text { UTI } \\
\text { Neuroci } \\
\text { rúrgica }\end{array}$ & $\begin{array}{l}\text { Descrever } \\
\text { alterações do } \\
\text { VMFSC em ACM } \\
\text { medido com } \\
\text { DTC, na posição } \\
\text { supina e e } \\
\text { sentada, em } \\
\text { PCTs adultos } \\
\text { Com HSA sem } \\
\text { VE ou com VEA. }\end{array}$ & $\begin{array}{l}\mathrm{N}=21 \\
\text { Sem } \\
\text { grupos }\end{array}$ & $\begin{array}{l}\text { Posição supina } \\
\text { com ELC de } 30^{\circ} \\
\text { e SD à beira do } \\
\text { leito com } \\
\text { avaliação das } \\
\text { variáveis HDN } \\
\text { (FC, PAS, PAD, } \\
\text { PAM) e VMFSC } \\
\text { em ACM nas } \\
\text { duas posições } \\
\text { (em supino e } \\
6 \text { min após SD) }\end{array}$ & $\begin{array}{lr}\text { Do total de } \\
\text { PCTs sem VE no } \\
\text { início do } \\
\text { estudo, } 5 \\
(31,2 \% \text { deles } \\
\text { geraram de EV } \\
\text { sintomas de dias } \\
\text { nos seguintes (que } \\
\text { correspondem } \\
\text { a } 23,8 \% \text { do } \\
\text { total de sujeitos } \\
\text { do estudo). }\end{array}$ & $\begin{array}{lr}\text { A mudança } & \text { de } \\
\text { supino para SD } & \text { SD } \\
\text { na HSA não gera } \\
\text { alterações HDN } \\
\text { significativas a } \\
\text { nível } \\
\text { cardiovascular ou } \\
\text { cerebral. } \\
\text { maioria dos casos } \\
\text { de VE foi em PCTs } \\
\text { com grau mais } \\
\text { elevado de HSA } \\
\text { (Fisher III e IV) e } \\
\text { nos dias de maior } \\
\text { risco de VE. } \\
\end{array}$ & $\begin{array}{l}\text { Amostra } \\
\text { pequena }\end{array}$ \\
\hline $\begin{array}{c}\text { Karic } 2015^{21} \\
\text { Noruega }\end{array}$ & $\begin{array}{l}\text { Prospect } \\
\text { ivo e } \\
\text { observa } \\
\text { cional }\end{array}$ & UTNI & $\begin{array}{lr}\text { Descrever } & \text { e } \\
\text { quantificar } & \text { conte da RP } \\
\text { conteúdo } \\
\text { adaptada a } \\
\text { PCTs com HSAa } \\
\text { aguda e avaliar } \\
\text { sua viabilidade }\end{array}$ & $\begin{array}{l}\mathrm{N}=37 \\
\text { Sem } \\
\text { grupos }\end{array}$ & $\begin{array}{l}\text { Posição no leito, } \\
\text { exercícios } \\
\text { passivos, } \\
\text { ativos, } \\
\text { transferências, } \\
\text { sedestação, } \\
\text { deambulação e } \\
\text { outros. }\end{array}$ & $\begin{array}{l}\text { Não foram } \\
\text { observados EA } \\
\text { graves para a } \\
\text { RP. }\end{array}$ & $\begin{array}{l}\text { A RP em PCTs } \\
\text { com HSAa é } \\
\text { viável desde o } 1^{\circ} \\
\text { dia após a CDA A } \\
\text { MB fora do leito } \\
\text { foi possível no } 3^{\circ} \\
\text { dia após CDA em } \\
\begin{array}{l}\text { PCTs com HSA de } \\
\text { grau menos } \\
\text { grave. }\end{array}\end{array}$ & $\begin{array}{l}\text { Amostra } \\
\text { pequena }\end{array}$ \\
\hline $\begin{array}{c}\text { Shah } 2018^{22} \\
\text { EUA }\end{array}$ & $\begin{array}{l}\text { Prospect } \\
\text { ivo e } \\
\text { observa } \\
\text { cional }\end{array}$ & UTNI & $\begin{array}{l}\text { Determinar a } \\
\text { segurança e a } \\
\text { viabilidade de } \\
\text { mobilidade em } \\
\text { PCTs de UTI } \\
\text { com DVE. }\end{array}$ & $\begin{array}{l}\mathrm{N}=90 \quad / \\
\text { Sem } \\
\text { grupos. } \\
\text { (Todos } \\
\text { recebera } \\
\mathrm{m} \quad \mathrm{PT} \\
\text { padrão da } \\
\text { UTI } \\
\text { incluindo } \\
\text { MP). }\end{array}$ & $\begin{array}{l}\text { Exercícios no } \\
\text { leito, } \\
\text { sedestação no } \\
\text { leito, } \\
\text { transferência } \\
\text { para cadeira até } \\
\text { marcha } \\
\text { estática, } \\
\text { caminhada com } \\
\text { e sem auxílio. }\end{array}$ & $\begin{array}{l}\text { Emese após } \\
\text { rolar um PCT } \\
\text { no leito; } \\
\text { Aumento da } \\
\text { PIC sem } \\
\text { alterações no } \\
\text { ExN; } \\
\text { Nenhuma } \\
\text { complicação } \\
\text { secundária de } \\
\text { HSA. }\end{array}$ & $\begin{array}{l}\text { Houve } 4(2,2 \%) \\
\text { eventos adversos } \\
\text { registrados } \\
\text { durante todo o } \\
\text { estudo }\end{array}$ & $\begin{array}{l}\text { Estudo } \\
\text { observacional } \\
\text {; houve viés } \\
\text { de coleta. }\end{array}$ \\
\hline
\end{tabular}

HSAa=Hemorragia Subaracnóidea aneurismática, HSA=Hemorragia Subaracnóidea, UTNI=Unidade de Terapia Neurointensiva, ENI=Enfermaria Neurointermediária, ELC=Elevaçã̃o da Cabeceira, ACM=Artéria Cerebral Média, VMFSC=Velocidade Média do Fluxo Sanguíneo Cerebral, DTC=Doppler Transcraniano, RL=Repouso no Leito, SD=Sedestação, VE=Vasoespasmo, VEA=Vasoespasmo Assintomático, $T \mathrm{TO}=$ Tratamento, $\mathrm{MB}=$ Mobilização, MP=Mobilização Precoce, RP=Reabilitação Precoce, $\mathrm{PCT}=$ Paciente, $\mathrm{PCTs}=$ pacientes, GC=Grupo Controle, GI=Grupo de Intervenção, $C D A=$ Correção do Aneurisma, TF=Terapia Física TO=Terapia Ocupacional, PT=Protocolo, AVDs=Atividades de Vida Diária, EA=Eventos Adversos, ExN=Exame Neurológico, HDN=hemodinâmicas, HIC=Hipertensão Intracraniana, MMII=Membros Inferiores, DVE=Derivação Ventricular Externa, FC=Frequência Cardíaca, PAS=Pressão Arterial Sistólica, PAD=Pressão Arterial Diastólica, PAM=Pressão Arterial Média. 
Tabela 3. Critérios de segurança e respectivas categorias com identificação das variáveis e parâmetros.

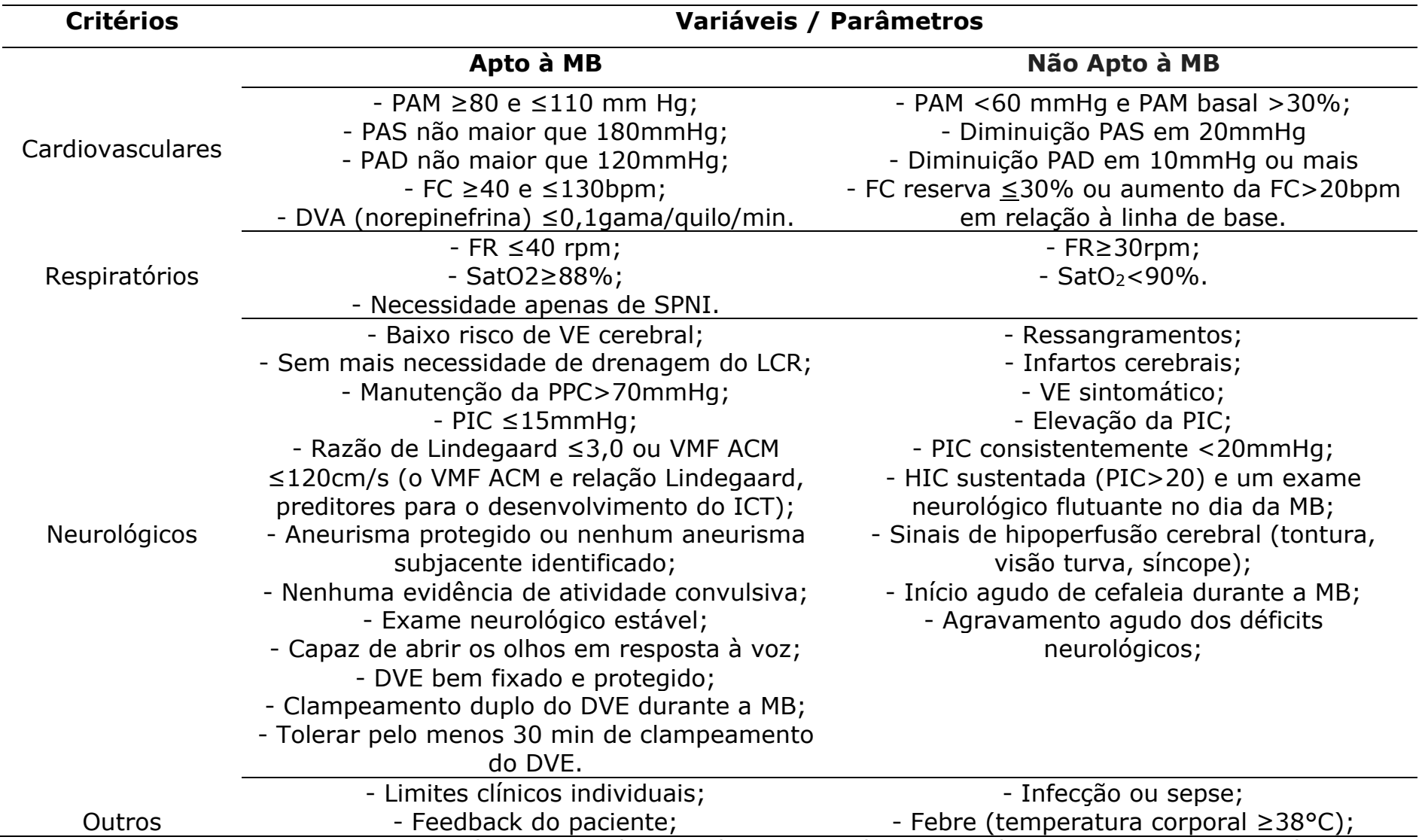

MB=Mobilização, PAM=Pressão Arterial Média, FC=Frequência Cardíaca, FR=Frequência Respiratória, PAS=Pressão Arterial Sistólica, PAD=Pressão Arterial Diastólica, PPC=Pressão de Perfusão Cerebral, PIC=Pressão Intracraniana, HIC=Hipertensão Intracraniana DVA=Droga Vasoativa, DVE=Derivação Ventricular Externa, LCR=Líquido Céfalo Raquidiano, ICT=Isquemia Cerebral Tardia , VMF=Velocidade Média do Fluxo, ACM=Artéria Cerebral Média, SPNI=Suporte Respiratório Não Invasivo, VE=Vasoespasmo.

Young $2019^{18}$ consideraram como critérios relativos para não iniciar a MB a presença de exame neurológico flutuante, instabilidade pulmonar ou cardiovascular e recusa do paciente, portanto, devendo ser reavaliados diariamente pela equipe com base no conhecimento da linha de base de cada paciente e das tendências fisiológicas durante sua internação.

Olkowski $2013^{19}$ estabeleceu como critérios: aneurisma protegido ou nenhum aneurisma subjacente identificado; Razão de Lindegaard (velocidade média da ACM/velocidade média da artéria carótida interna) $\leq 3,0$ ou Velocidade Média 
do Fluxo Sanguíneo da Artéria Cerebral Media (VMF ACM) $\leq 120 \mathrm{~cm} / \mathrm{s}$; PAM $\geq 80$ e $\leq 110 \mathrm{mmHg}$, ou seja, com menor risco de Isquemia Cerebral Tardia (ICT); FC $\geq 40$ e $\leq 130$ bpm; FR $\leq 40$ rpm; oximetria de pulso $\geq 88 \%$; PIC $\leq 15 \mathrm{mmHg}$; nenhuma evidência de atividade convulsiva; exame neurológico estável; capaz de abrir os olhos em resposta à voz e capacidade de mover uma extremidade sob comando. O programa foi viável logo após a correção do aneurisma e até a alta hospitalar e os EA que ocorreram foram mínimos (em 5,9\%; 17 de 286 sessões) e atribuídos às alterações fisiológicas normais associadas à atividade ${ }^{19}$. A taxa de EA neste estudo $(5,9 \%)$ é comparável àquela em outros estudos de $\mathrm{MB}^{18,23}$.

O controle da PIC como critério de segurança é unanime, justifica-se pelo fato de que seu aumento acentuado diminui a PPC e resulta em isquemia do tronco encefálico, com redução da pressão parcial de oxigênio e liberação de neurotransmissores, que induzem a excitação do centro simpático, mecanismos que podem estar associados ao desenvolvimento de VE cerebral ${ }^{16,24}$. Portanto, a prevenção de ICT e HIC, e uma PPC adequada são indispensáveis no cuidado com pacientes com $\mathrm{HSAa}^{19}$. O estudo de Moyer $2017^{17}$ não usou a ICT como uma contraindicação absoluta para a MB, entretanto, o paciente era reavaliado diariamente e a presença de flutuações no quadro neurológico era investigada descartando febre, infecção ou administração de medicamentos como causa. 
Dispositivos e padrões de rotina podem ser barreira ou não para o início da $\mathrm{MB}^{25}$. No estudo de Young $2019^{18}$ os pacientes incluídos realizavam eletroencefalografia contínua, o que não impediu a participação no programa de $M B$, a menos que houvesse convulsão ou alguma evidência de isquemia. Da mesma forma, ventilação mecânica (VM), drenos ventriculares ou lombares e linhas arteriais não impediram a participação. Já o estudo de Shah $2018^{22}$ não inclui paciente em VM por considerar que, na unidade em estudo, a maioria dessa população alcança menor pontuação na Escala de Coma de Glasgow (ECG) o que poderia interferir na identificação do estado de confusão mental que foi avaliado pelo Método de Avaliação de Confusão para Unidade de Cuidados Intensivos (CAM-ICU) e usado como critério de exclusão. Em Young 201918, a prática da instituição era enviar pacientes com HSA para radiologia nas primeiras 24 horas de admissão com repouso subsequente, só após a sua suspensão os pacientes eram elegíveis para MB.

Além de aspectos inerentes a rotinas de setor, presença de dispositivos invasivos ${ }^{25}$ de neuromonitoramento podem impedir a MB precoce, seja por medo de deslocamento de cateteres, hemorragia ou drenagem inadequada do LCR, dentre outras razões 22,25 . Porém, estudos ${ }^{17,18,22}$ apontam e utilizam estratégias como, por exemplo, fazer graduação nas etapas de protocolos de MB; garantir a fixação segura de linhas, drenos e tubos, dentre outros, com um trabalho em equipe interdisciplinar e definições de papeis dos integrantes ${ }^{25}$. Com relação a prevenir deslocamento 
acidentais de drenos, Shah $2018^{22}$ implementou uma lista de verificação de segurança, a MB só iniciava após checagem de seus itens. Após essa iniciativa, não houve nenhum deslocamento de cateter ou complicações de sangramento foram atribuíveis ao uso do protocolo de MBP. Ainda em relação ao uso de drenos, em um dos estudos ${ }^{18}$ os pacientes foram excluídos da MB se não conseguissem tolerar pelo menos 30 minutos de clampeamento da Derivação Ventricular Externa (DVE) em repouso no leito e para evitar a drenagem inadequada do LCR, o protocolo exigia clampeamento duplo do DVE enquanto o paciente estava fora da cama (com uma ordem, as enfermeiras poderiam drenar uma determinada quantidade de LCR a cada hora). Moyer $2017^{17}$ também utilizaram da mesma estratégia de pinçamento duplo. Ambos os estudos mostraram que era viável mobilizar de forma segura o paciente com DVE, desde que, tomando as devidas precauções ${ }^{17,18}$.

Quanto ao momento ideal para começar a MB, considerando que o VE cerebral ocorre a partir do $3^{\circ}$ pós ictus e pode atingir o pico entre o 70 e o $10^{\circ}$ dia após HSA ${ }^{15,26}$ e que o período de maior risco de ressangramento é entre duas a quatro semanas após ictus ${ }^{5}$ e sabendo que estas são algumas das razões pelas quais, historicamente, os paciente são mantidos em repouso absoluto, vemos no estudo de Karic $2016^{15}$ que escolheram o nível de MB alcançado nos dias 4 e 7 ao comparar o grupo com tratamento padrão da unidade e o grupo que recebeu MB precoce. Notou-se que em média, a participação no PMB precoce começou em 3,2 
dias após HSAa, os pacientes necessitaram de 5,4 dias para participar da atividade fora do leito e de 10,7 dias para caminhar $\geq 15,24 \mathrm{~m}$ e não houve prejuízos atribuídos ao programa. Da mesma forma, o estudo de Karic $2015^{21}$ usando um mesmo algoritmo de MB mostrou que a reabilitação precoce nos pacientes com HSAa é viável desde o $1^{\circ}$ dia após a correção do aneurisma (CDA) e a atividade fora do leito foi possível no $3^{\circ}$ dia após a CDA em casos de HSAa de grau menos grave. Já Merino $2014^{20} \mathrm{com}$ o objetivo de descrever alterações da VMF ACM com DTC e nas variáveis hemodinâmicas (HDN), na posição supina e sentada, em pacientes adultos com HSA sem VE cerebral ou com VE assintomático viram que, do total de sujeitos sem VE no início do estudo, $5(31,2 \%)$ deles geraram sintomas de EV nos dias seguintes ${ }^{20}$. A maioria desses casos foi em pacientes com grau mais elevado de HSA (Fisher III e IV) ${ }^{27}$ e foram sentados no período de maior risco de VE (em média no $7^{\circ}$ dia de HSA). No entanto, nenhum deles apresentou alterações significativas nas variáveis HDN ou da VMF ACM no dia da medição, nem sintomas de VE nos dias seguintes até $021^{\circ}$ de $\mathrm{HSA}^{20}$. Contudo a amostra desse estudo foi pequena e descreve as mudanças na VMF ACM associadas a sentar em um único momento, os resultados, portanto, devem ser interpretados com cautela.

Investigando consequências de mudanças de posição em pacientes com HSAa, estudos anteriores ${ }^{28,29}$ viram que a posição da cabeceira da cama não aumentou a incidência de ICT ou alterou a HDN cerebral. Também viram que exercícios 
passivos e ativos em pacientes internados em unidade intensiva neurocirúrgica não aumentaram PIC ou a PPC 19,28,29. Nos estudos em que ocorreram EA relacionados a PAM abaixo do limiar $(70 \mathrm{mmHg})$ os pacientes estavam em treinamento de transição para uma posição mais vertical (supino para elevação de $30^{\circ}$ da cabeceira, daí para sentado e de sentado para em pé $)^{19,29}$. Tais achados sugerem que essas transições posicionais devem ser progressivas e com monitoramento da PAM principalmente se o paciente está em um momento em que manter a sua PPC adequada é uma prioridade, ou seja, em pacientes com maior risco de $\mathrm{ICT}^{19}$.

Considerando todas os aspectos supracitados, com 0 intuito de reduzir as complicações decorrentes da restrição prologada ao leito, a MB dos pacientes com HSAa deve iniciar logo que estes alcancem um quadro de estabilidade ${ }^{16,30}$. Para isto, o neuromonitoramento adequado é imprescindível na interpretação do quadro clínico e na então elegibilidade correta para $\mathrm{MBB}^{19}$. Apesar de alguns dos estudos não exporem com clareza os critérios utilizados, os EA ocorridos e o protocolo de MB aplicado, foi possível identificar que o cerne da MB nesse perfil de pacientes foi a progressão gradual das etapas de MB.

Podemos relatar algumas limitações na realização da presente revisão como, número reduzido de estudos que puderam ser incluídos e ainda com agravante da baixa qualidade metodológica, pois, dos estudos de intervenção passiveis de avaliação pela Escala Pedro, não tivemos nenhum estudo de alta qualidade e apenas 3 de qualidade 
intermediária. Tivemos como desafio a dificuldade na comparação dos resultados dos estudos devido a variação de desfechos analisados, e ainda pela heterogeneidade dos sujeitos (pacientes com HSAa com aneurisma corrigido ou não, aneurisma tratado com terapia endovascular ou outra, HSAa em população de idosos, diferentes graus de HSAa pela escala de Fisher ${ }^{27}$ ), dentre outros. E tendo em vista a escassez de estudos abordando o tema em questão, consideramos incluir artigos, mesmo de baixo nível de evidência. Portanto, ressaltamos que os resultados encontrados fornecem a nossa pergunta norteadora uma resposta a qual deve ser complementada por estudos futuros com amostras maiores e métodos mais adequados.

\section{CONCLUSÃO}

Os critérios de segurança para o início da fisioterapia motora em pacientes com HSAa foram sumarizados nas principais categorias: neurológica, cardiovascular e respiratória. Na categoria neurológica foi onde tivemos maior número de itens com variáveis a serem consideradas para a elegibilidade do paciente a iniciar a MB. Em suma, a MB do paciente com HSAa deve, idealmente, começar assim que ele estiver estabilizado, a fim de reduzir as complicações do repouso prolongado no leito. Os critérios de segurança se concentraram em garantir a estabilidade neurológica e fisiológica antes do início das primeiras sessões de MB. E o cerne da mobilização nesse perfil de pacientes foi o aumento gradual no nível de MB levando em consideração a presença 
e o grau de VE cerebral, barreiras impostas por rotinas de setor e/ou por dispositivos invasivos e, acima de tudo, o monitoramento adequado de todas as variáveis que implicam na interpretação correta de possíveis alterações do quadro clínico e neurológico nessa população. Destacamos a necessidade de mais estudos experimentais nessa área com qualidade metodológica adequada e com populações mais especificas a fim de fornecer resposta segura e de evidência incontestável e com maior aplicabilidade na prática clínica.

\section{REFERÊNCIAS}

1.Coelho IGBSA, Costa JMD, Silva EIPA. Hemorragia subaracnóidea espontânea não aneurismática: perimesencefálica versus não perimesencefálica. Rev Bras Terap Intensiva 2016;28:141-6. https://doi.org/10.5935/0103-507X.20160028

2.Guaresi JR, Iung TC, Branco LTO, Medeiros MS, Sakae TM. Sequelas em pacientes com hemorragia subaracnóidea por ruptura de aneurisma intracraniano. Arq Catarin Med 2011;40:34-40.

http://www.acm.org.br/acm/revista/pdf/artigos/860.pdf

3.Van Gijn J, Kerr RS, Rinkel GJ. Subarachnoid haemorrhage. Lancet 2007;369:306-18. https://doi.org/10.1016/S0140-6736(07)60153-6

4.Stiller K. Safety issues that should be considered when mobilizing critically ill patients. Crit Care Clin 2007;23:35-53. https://doi.org/10.1016/j.ccc.2006.11.005

5. Ma Z, Wang Q, Liu M. Early versus delayed mobilisation for aneurysmal subarachnoid haemorrhage. Cochr Datab Syst Ver 2013; 5:CD008346.

https://doi.org/10.1002/14651858.CD008346.pub2

6. Pontes-Neto OM, Oliveira-Filho J, Valiente R, Friedrich M, Pedreira B, Rodrigues $B C B$, et al. Diretrizes para o manejo de pacientes com hemorragia intraparenquimatosa cerebral espontânea. Arq Neuropsiquiatr 2009;67:940-50. https://doi.org/10.1590/S0004282X2009000500034

7.AVERT Trial Collaboration group. Efficacy and safety of very early mobilisation within $24 \mathrm{~h}$ of stroke onset (AVERT): a randomised controlled trial. Lancet 2015;386:46-55.

https://doi.org/10.1016/S0140-6736(15)60690-0

8.Conceição TMA da, Gonzáles AI, Figueiredo FCXS de, Vieira DSR, Bündchen DC. Critérios de segurança para iniciar a mobilização precoce em unidades de terapia intensiva. Revisão sistemática. Rev Bras Ter Intensiva 2017;29:509-19.

https://doi.org/10.5935/0103- 
9.Luque A, Gimenes AC. Reabilitação precoce em terapia intensiva. Pneumol Paul 2013;27:44-8.

https://www. researchgate.net/publication/261019287 Reabilitacao P recoce em Terapia intensiva

10.Steiner T, Juvela S, Unterberg A, Jung C, Forsting M, Rinkel G, et al. European Stroke Organization guidelines for the management of intracranial aneurysms and subarachnoid haemorrhage. Cerebrovasc Dis 2013;35:93-112. https://doi.org/10.1159/000346087

11. Moher D, Liberati A, Tetzlaff J, Altman DG, PRISMA Group. Preferred reporting items for systematic reviews and meta-analyses: the PRISMA statement. Ann Intern Med 2009;151:264-9.

https://doi.org/10.7326/0003-4819-151-4-200908180-00135

12. Maher CG, Sherrington C, Herbert RD, Moseley AM, Elkins M. Reliability of the PEDro scale for rating quality of randomized controlled trials. Phys Ther 2003;83:713-21.

https://doi.org/10.1093/PTJ/83.8.713

13.Bündchen DC, Gonzáles AI, Noronha M, Brüggemann AK, Sties SW, Carvalho T. Ventilação não invasiva e tolerância ao exercício na insuficiência cardíaca: uma revisão sistemática e metanálise. Braz J Phys Ther 2014;18:385-94. https://doi.org/10.1590/bjptrbf.2014.0039

14.Karic T, Røe C, Nordenmark TH, Becker F, Sorteberg W, Sorteberg A. Effect of early mobilization and rehabilitation on complications in aneurysmal subarachnoid hemorrhage. J Neurosurg 2017;126:518-26. https://doi.org/10.3171/2015.12.JNS151744

15.Karic T, Røe C, Nordenmark TH, Becker F, Sorteberg A. Impact of early mobilization and rehabilitation on global functional outcome one year after aneurysmal subarachnoid hemorrhage. J Rehabil Med 2016;48:676-82. https://doi.org/10.2340/16501977-2121

16. Rocca A, Pignat J-M, Berney L, Jöhr J, Van de Ville D, Daniel RT, et al. Sympathetic activity and early mobilization in patients in intensive and intermediate care with severe brain injuries: a preliminary prospective randomized study. BMC Neurol 2016;16:169. https://doi.org/10.1186/s12883-016-0684-2

17. Moyer M, Young B, Wilensky EM, Borst J, Pino W, Hart M, et al. Implementation of an Early Mobility Pathway in Neurointensive Care Unit Patients With External Ventricular Devices. J Neurosci Nurs 2017;49:102-7. https://doi.org/10.1097/JNN.0000000000000258

18. Young B, Moyer M, Pino W, Kung D, Zager E, Kumar MA. Safety and Feasibility of Early Mobilization in Patients with Subarachnoid Hemorrhage and External Ventricular Drain. Neurocrit Care 2019;31:88-96. https://doi.org/10.1007/s12028-019-00670-2

19. Olkowski BF, Devine MA, Slotnick LE, Veznedaroglu E, Liebman KM, Arcaro $\mathrm{ML}$, et al. Safety and feasibility of an early mobilization program for patients with aneurysmal subarachnoid hemorrhage. Phys Ther 2013;93:208-15. https://doi.org/10.2522/ptj.20110334

20.Merino C, Heap P, Vergara V, Yáñez A, Rivera R. Descripción de los cambios en la velocidad media de flujo sanguíneo cerebral en posición 
supino y sedente, en pacientes con hemorragia subaracnoidea aneurismática con vasoespasmo asintomático o sin vasoespasmo: Serie de casos. Rev Med Chile 2014;142:1502-9. https://doi.org/10.4067/S0034-98872014001200002

21.Karic T, Sorteberg A, Haug Nordenmark T, Becker F, Roe C. Early rehabilitation in patients with acute aneurysmal subarachnoid hemorrhage. Disabil Rehabil 2015;37:1446-54. https://doi.org/10.3109/09638288.2014.966162

22.Shah SO, Kraft J, Ankam N, Bu P, Stout K, Melnyk S, et al. Early Ambulation in Patients With External Ventricular Drains: Results of a Quality Improvement Project. J Intensive Care Med 2018;33:370-4. https://doi.org/10.1177/0885066616677507

23.PohIman MC, Schweickert WD, Pohlman AS, Nigos C, Pawlik AJ, Esbrook CL, et al. Viabilidade da terapia física e ocupacional a partir do início da mecânica. Crit Care Med 2010;38:2089-94. https://doi.org/10.1097/CCM.0b013e3181f270c3

24. Miller BA, Turan N, Chau M, Pradilla G. Inflammation, vasospasm, and brain injury after subarachnoid hemorrhage. Biomed Res Int 2014;2014:384342. https://doi.org/10.1155/2014/384342

25.Dubb R, Nydahl P, Hermes C, Schwabbauer N, Toonstra A, Parker AM, et al. Barriers and Strategies for Early Mobilization of Patients in Intensive Care Units. Ann Am Thorac Soc 2016;13:724-30. https://doi.org/10.1513/AnnalsATS.201509-586CME

26. Heros RC, Zervas NT, Varsos V. Cerebral vasospasm after subarachnoid hemorrhage: an update. Ann Neurol 1983;14:599-608. https://doi.org/10.1002/ana.410140602

27. Oliveira AMP, Paiva WS, Figueiredo EG, Oliveira HA, Teixeira MJ. Fisher revised scale for assessment of prognosis in patients with subarachnoid hemorrhage. Arq Neuropsiquiatr 2011;69:910-3. https://doi.org/10.1590/S0004-282X2011000700012

28.Zhang Y, Rabinstein AA. Lower head of the bed position does not change blood flow velocity in subarachnoid hemorrhage. Neurocrit Care 2011;14:73-6. https://doi.org/10.1007/s12028-010-9444-0

29.Blissitt PA, Mitchell PH, Newell DW, Woods SL, Belza B. Cerebrovascular dynamics with head-of-bed elevation in patients with mild or moderate vasospasm after aneurysmal subarachnoid hemorrhage. Am J Crit Care 2006;15:206-16.

https://doi.org/10.4037/ajcc2006.15.2.206

30.Titsworth WL, Hester J, Correia T, Reed R, Guin P, Archibald L, et al. The effect of increased mobility on morbidity in the neurointensive care unit. J Neurosurg 2012;116:1379-88.

https://doi.org/10.3171/2012.2.JNS111881 